\title{
Selection of Fluorescent, Bioluminescent and
}

\author{
Radioactive Tracers to Accurately Reflect
}

\section{Extracellular Vesicle Biodistribution in Vivo}

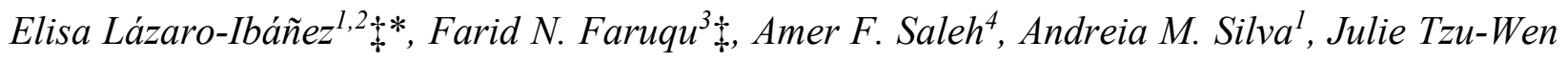
Wang ${ }^{3}$, Janusz Rak ${ }^{5}$, Khuloud T. Al-Jamal ${ }^{3}$, Niek Dekker ${ }^{l}$ *

${ }^{1}$ Discovery Biology, Discovery Sciences, BioPharmaceuticals R\&D AstraZeneca, Gothenburg, 43150, Sweden. ${ }^{2}$ Advanced Drug Delivery, Pharmaceutical Sciences, BioPharmaceutical R\&D, AstraZeneca, Gothenburg, 43150, Sweden. ${ }^{3}$ Institute of Pharmaceutical Science, School of Cancer \& Pharmaceutical Sciences, King's College London, London, SE1 9NH, United Kingdom. ${ }^{4}$ Functional and Mechanistic Safety, Clinical Pharmacology \& Safety Sciences, BioPharmaceuticals R\&D AstraZeneca, Cambridge, CB2 0AA, United Kingdom. ${ }^{5}$ Research Institute of the McGill University Health Centre, Glen Site, McGill University, Montreal, Quebec, H4A 3J,1 Canada.

*To whom correspondence should be addressed:

Niek Dekker: E-mail: niek.dekker@astrazeneca.com; Elisa Lázaro-Ibáñez: E-mail: elisa.lazaroibanez@astrazeneca.com, Khuloud Al-Jamal: E-mail: khuloud.al-jamal@kcl.ac.uk. 
A

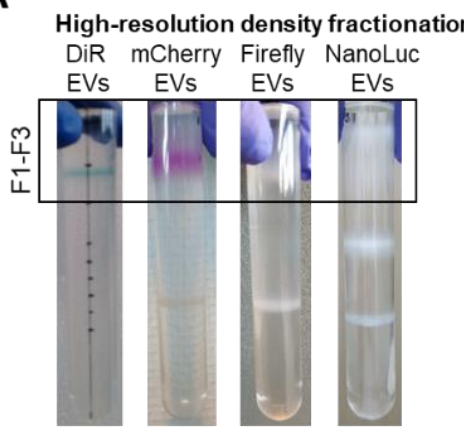

\section{C}

TEM analysis of high-density fractions

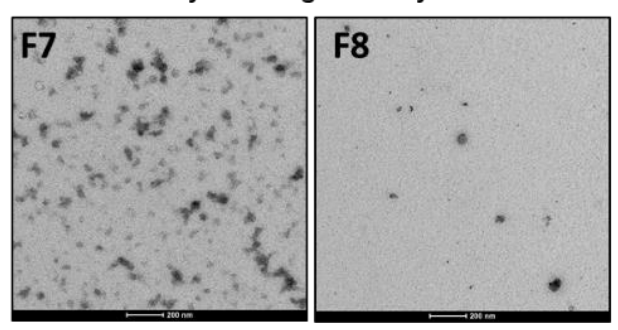

B

Size analysis of low-density fractions

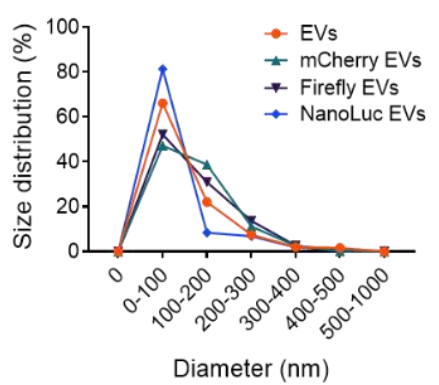

D

TEM analysis of low density fractions

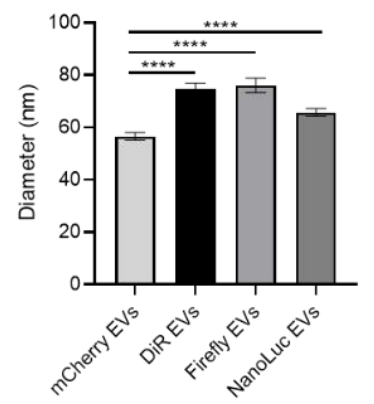

Figure S1. In vitro characterization of genetically engineered and membrane-labeled EVs. (A) Representative images of high-resolution iodixanol density gradients for the separation of low-density EV fractions (corresponding to F1-F3). (B) Size determination of DiR-labeled EVs and mCherry- Firefly- and NanoLuc-engineered EVs from low-density fractions. Fraction 1 to 3 were pooled and analyzed by Nanoparticle Tracking Analyzer. A representative graph showing the normalized size distribution of EVs based on their size range (nm) for the different EV types is shown. (C) Representative Transmission Electron Microscopy (TEM) micrographs of high-density fractions from CD63 overexpressing cells. Scale bars $200 \mathrm{~nm}$. (D) Image analysis of TEM micrographs to evaluate the diameter in nanometers $(\mathrm{nm})$ of the different EV preparations from low-density fractions. A total of 175 images were analyzed. Results are expressed as mean \pm SEM. Non-parametric Kruskal-Wallis multiple comparison test. ****p value $<0.0001$. 
A

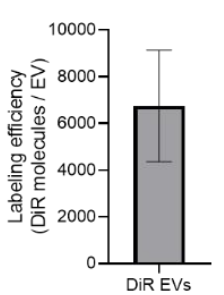

B

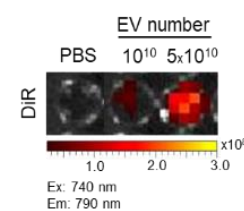

D

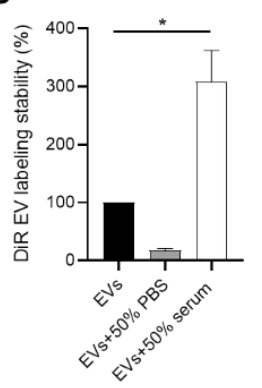

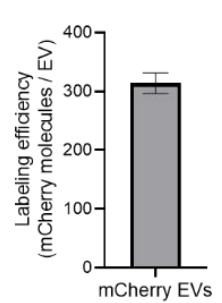
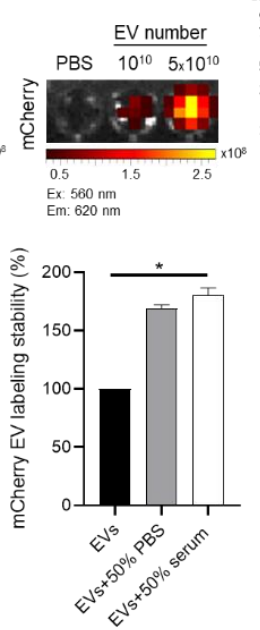

C

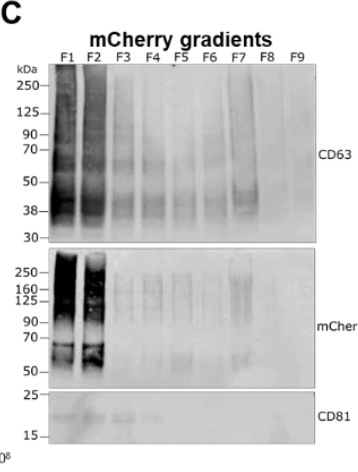

E

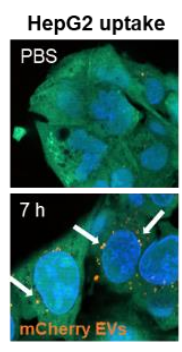

$\mathbf{F}$
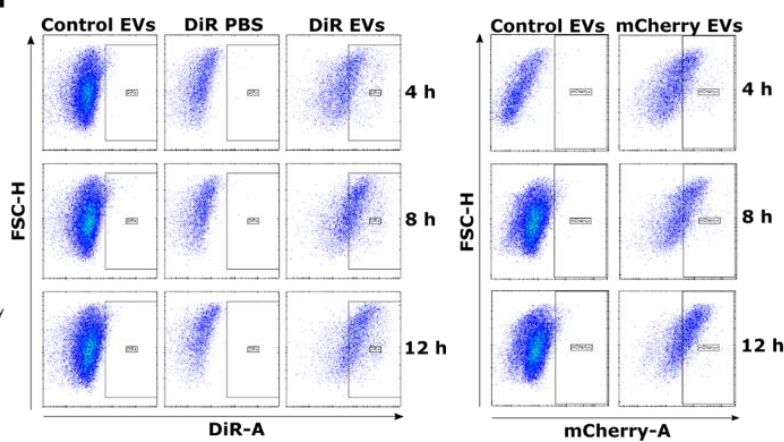

G

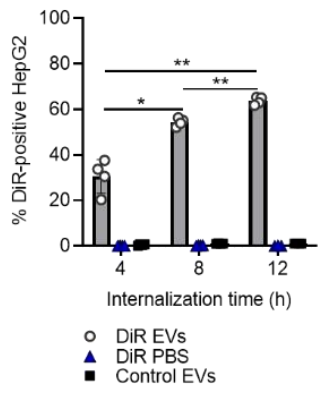

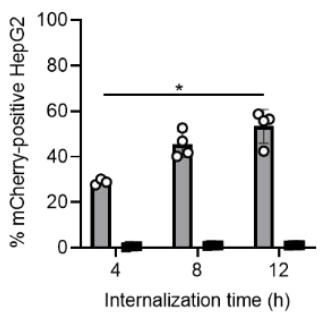

- mCherry EVs

Figure S2. Labeling efficiency, stability and in vitro detection of fluorescent EVs. (A) Labeling efficiency of DiR and mCherry EVs represented as the number of molecules per particle after EV bulk fluorescent analysis. Graphs show the mean values \pm SEM of three independent samples. (B) Representative fluorescent plate readouts of mCherry EVs and DiR-labeled EVs corresponding to $10^{10}$ and $5 \times 10^{10}$ particles, respectively (10 and $50 \%$ of the in vivo injected dose). The same volume of PBS was used as control. EV samples were imaged with mCherry filter (Ex: 560 nm / Em: $620 \mathrm{~nm}$ ) or DiR filter (Ex: 740 nm / Em: $790 \mathrm{~nm}$ ). (C) Representative Western blotting analysis of (F1-F9) density fractions of the mCherry-CD63 engineered EVs (12 $\mu \mathrm{L} /$ each). Membranes were blotted with CD63, CD81, and mCherry antibodies. (D) Stability of DiR-labeled EVs and mCherry EVs in serum. Equal numbers of EVs were assayed fresh or incubated with either $50 \%$ PBS or $50 \%$ serum at $37{ }^{\circ} \mathrm{C}$ for $24 \mathrm{~h}$ before fluorescent readouts. The fluorescent signals obtained were expressed as \% signal relative to that of the fresh DiR EVs or mCherry EVs. Values are represented as the mean \pm SEM. One-way ANOVA with Kruskal-Wallis multiple comparisons test. * $p$ value $<0.05$. (E) In vitro uptake of mCherry EVs by HepG2 cells by live confocal microscopy. Cells were treated with increasing concentrations of mCherry EVs to visualize their intracellular accumulation after their internalization by the liver cells. Representative images of HepG2 cells incubated with PBS as Ctrl or 5000 mCherry EVs per cell at 7 $\mathrm{h}$ are shown. mCherry EVs are shown in the red channel, Hoechst nuclei staining is shown in blue and Cell Tracker staining is shown in green. 60x magnification of all images. (F) In vitro cell uptake analysis of DiR-labeled EVs and 
mCherry EVs measured by flow cytometry. HepG2 cells were incubated with DiR-labeled EVs (DiR EVs), DiRlabeled PBS control (DiR PBS), mCherry EVs and non-labeled EVs (Control EVs) for up to $12 \mathrm{~h}$. Representative dot plots of HepG2 positive cells at $4 \mathrm{~h}, 8 \mathrm{~h}$ and $12 \mathrm{~h}$ post-EV treatment are shown. (E) Quantification of the percentage of DiR-positive and mCherry-positive HepG2 cells at all timepoints. $\mathrm{N}=3$. Values in all graphs are expressed as mean \pm SEM and are corrected for background signal from non-stimulated cells. $* p$ value $<0.05$, **p value $<0.005$, as determined by Two-way ANOVA, followed by Sidak's multiple comparison test.

A

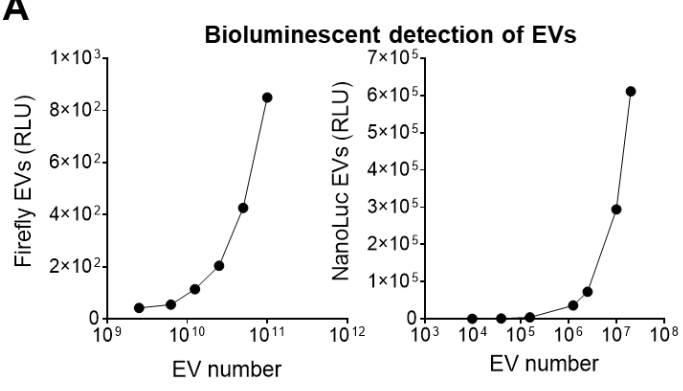

B

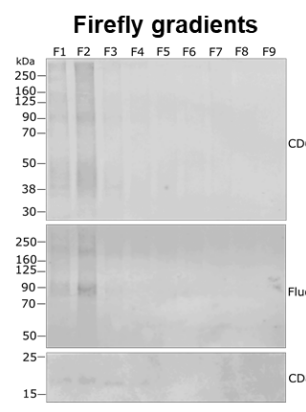

C

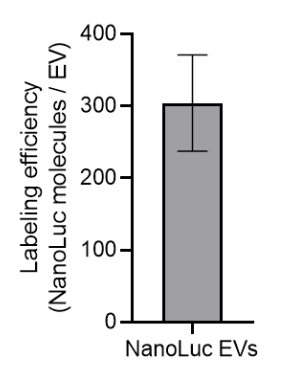

D
E

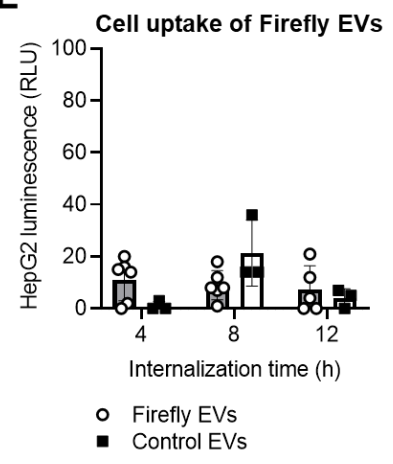

$\mathbf{F}$
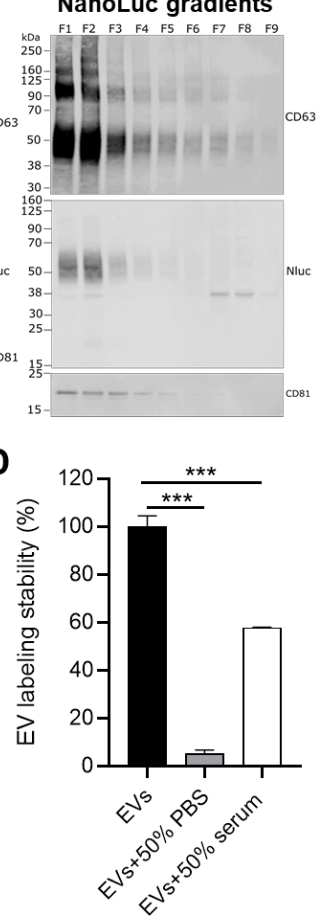

G

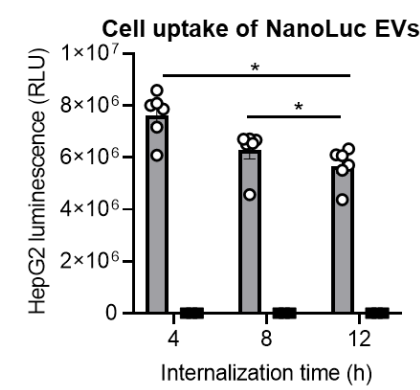

- NanoLuc EVs

- Control EVs

Membrane radiolabeling of EVs

\begin{tabular}{cc}
\hline EVs & $\begin{array}{c}\text { Radiolabeling efficiency } \\
(\%)\end{array}$ \\
\hline Expi293F & $73.5 \pm 4.7$
\end{tabular}

${ }^{1}$ Values are expressed as mean $\pm S D$, where $n=3$

Radiolabeling stability of ${ }^{111}$ Indium-DTPA EVs
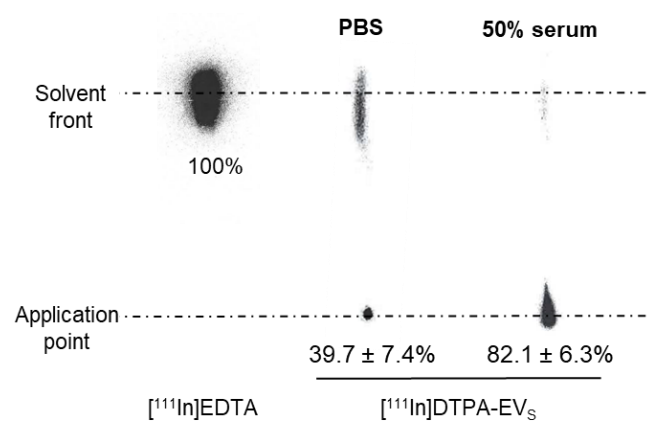

Figure S3. Labeling efficiency, stability and in vitro detection of bioluminescent and radiolabeled EVs. (A) Representative in vitro bioluminescence readouts (RLU) of Firefly EVs ( $3 \times 10^{9}-1 \times 10^{11}$ particles) and NanoLuc EVs $\left(9 \times 10^{3}-2 \times 10^{7}\right.$ particles) after the addition of D-luciferin and furimazine substrates. (B) Representative Western blotting analysis of (F1-F9) density fractions from Firefly-CD63 and NanoLuc-CD63 engineered EVs (12 $\mu$ L/each). 
Membranes were blotted with CD63, CD81, Firefly and NanoLuc antibodies. (C) Labeling efficiency of NanoLuc EVs is represented as the average number of NanoLuc molecules per EV. Analysis was based on comparative Western blots of CD63-mCherry and CD63-NanoLuc samples. Bar graph shows the mean \pm SEM or three independent experiments. (D) Stability of NanoLuc EVs in serum. Equal numbers of NanoLuc EVs (3 x $10^{6}$ particles) were either directly subjected to the NanoGlo luciferase assay or incubated with either $50 \%$ PBS or $50 \%$ serum at $37{ }^{\circ} \mathrm{C}$ for 24 $\mathrm{h}$ before the bioluminescent assay. The bioluminescence signals obtained were expressed as $\%$ activity relative to that of the fresh NanoLuc EV sample. Values are expressed as the mean \pm SEM. One-way ANOVA with Dunnett's multiple comparisons test. $* * * p$ value $<0.005$. (E) Analysis of the in vitro cell uptake of Firefly EVs and NanoLuc EVs measured by bioluminescence. HepG2 cells were incubated with bioluminescent EVs and non-labeled EVs (Control EVs) for up to $12 \mathrm{~h}$. Cells were incubated with the substrates D-luciferin or furimazine before the readout. Representative graphs of HepG2 positive cells at $4 \mathrm{~h}, 8 \mathrm{~h}$ and $12 \mathrm{~h}$ post-EV treatment are shown. ${ }^{*} p$ value $<0.05$ as determined by Two-way ANOVA, followed by Sidak's multiple comparison test. (F) Radiolabeling efficiency in (\%) of Expi293F EVs. Values are expressed as the mean \pm SD ( $n=3)$. (G) Radiochemical stability of membrane-labeled Expi293F EVs. Radiolabeled EVs ([111 In]-DTPA EVs) were incubated in either PBS or $50 \%$ serum for $24 \mathrm{~h}$ at $37^{\circ} \mathrm{C}$ and then spotted on a thin layer chromatography paper. The paper was then run on $0.1 \mathrm{M}$ ammonium acetate with 0.25 mM EDTA (pH 5.5) as the mobile phase and imaged using a phosphor imager. Radiochemical stability was calculated as the percentage of radioactivity remaining at the application point. Values are expressed as mean $\pm \mathrm{SD}$, where $\mathrm{n}=3$. 
A

Real-time in vivo imaging

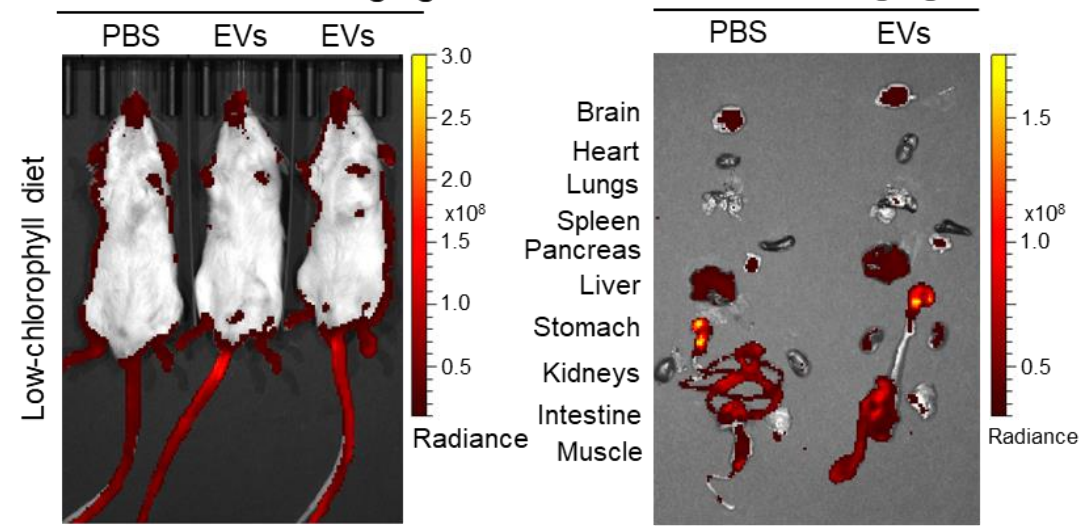

B

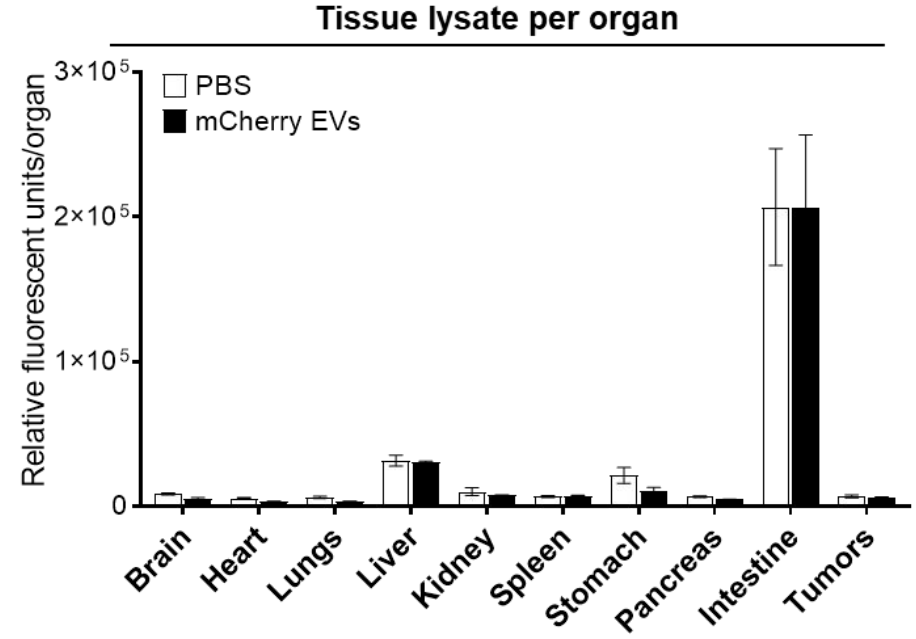

Figure S4. In vivo evaluation of mCherry Expi293F EVs in mice fed an alfalfa-free diet (low-chlorophyll).

Animals were put on this diet for a week before the imaging to reduce background fluorescence. (A) Representative real-time in vivo live imaging of tumor-bearing $\mathrm{BALB} / \mathrm{c}$ mice intravenously injected with $1 \times 10^{11}$ mCherry EVs via the tail vein at $24 \mathrm{~h}$ post-administration. Following in vivo imaging of the animals, major organs were excised and $e x$ vivo imaged. Representative ex vivo images of organs from PBS-treated and EV-treated animals are shown. (B) CT26 tumor-bearing BALB/c mice were intravenously injected with $10^{11}$ mCherry EVs or PBS via the tail vein. Organs were homogenized using a lysis buffer and cleared of tissue debris before mCherry fluorescence detection using the IVIS ${ }^{\circ}$ Lumina III system. Fluorescence signals are expressed per organ. PBS-treated and EV-treated animals are shown. Values are represented as mean \pm SEM, where $n=3$ for each group. 


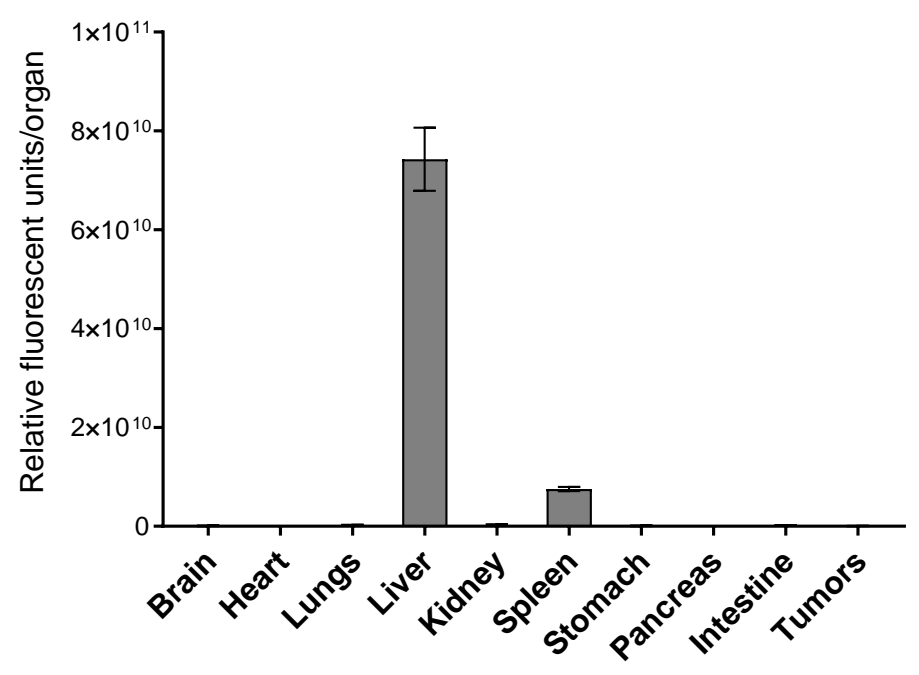

Figure S5. Organ biodistribution profile of DiR-labeled EVs in subcutaneous CT26 tumor-bearing BABL/c mice. Mice were injected with $1 \times 10^{11}$ DiR-labeled EVs or PBS and sacrificed $24 \mathrm{~h}$ post-injection. Mice were perfused with saline, and major organs were excised for fluorescent analysis. Organs were homogenized using a lysis buffer and cleared of tissue debris prior to DiR fluorescence detection using the IVIS® Lumina III system. Fluorescence signals are expressed per gram organ. DiR values were normalized by the organ values of the PBS control animals. The graph shows relative fluorescent units per organ represented as mean \pm SEM, where $n=3$ for each group. 
A

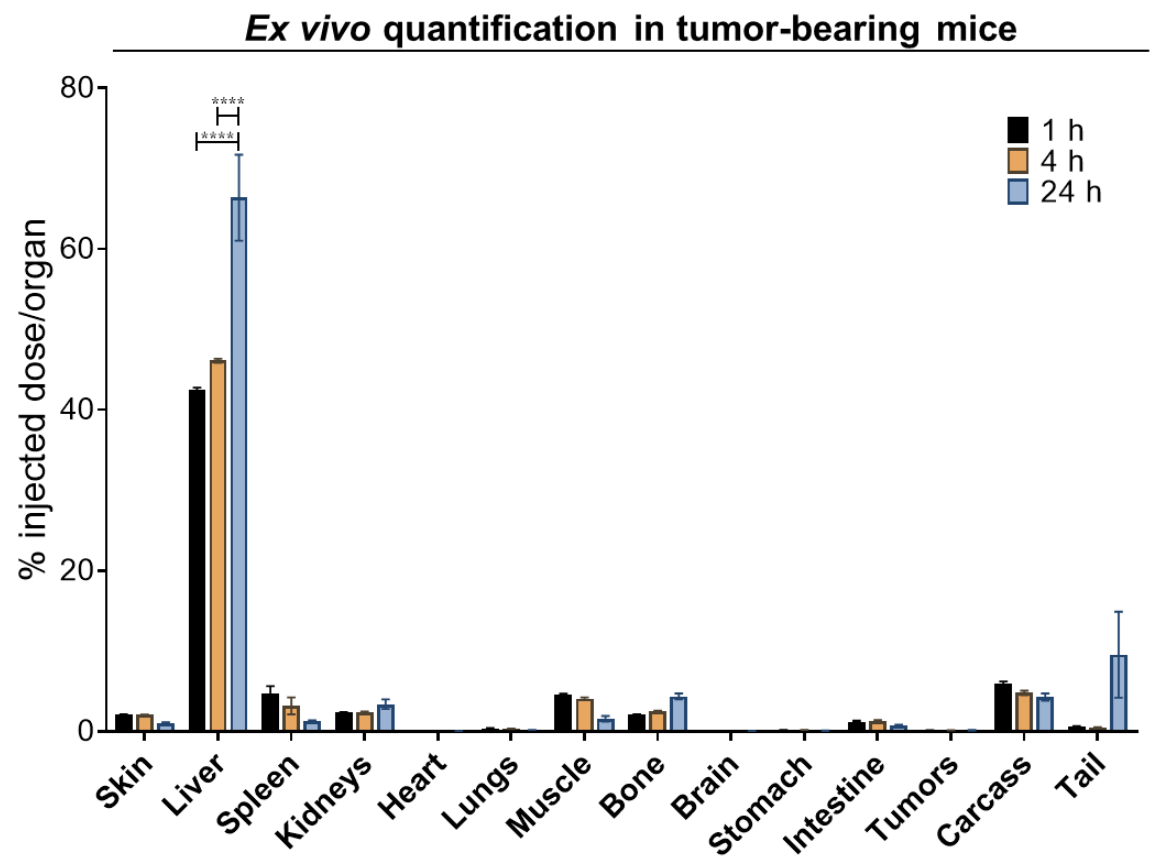

B

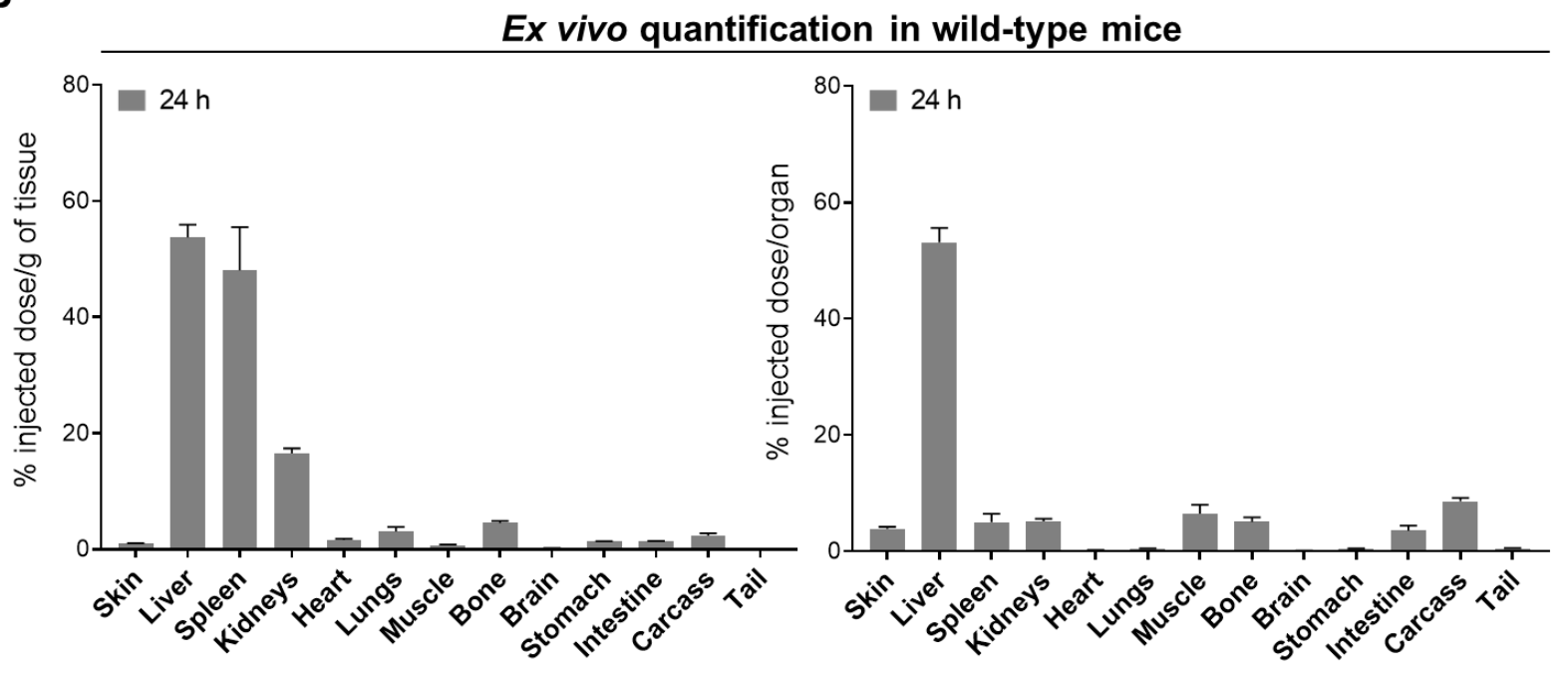

Figure S6. Ex vivo organ biodistribution of radiolabeled ${ }^{111}$ Indium-DTPA Expi293F EVs in mice. (A) Membrane-radiolabeled Expi293F EVs were intravenously administered into subcutaneous CT26 tumor-bearing BALB/c mice at a dose of $1 \times 10^{11}$ radiolabeled ${ }^{111}$ Indium vesicles ([ $\left.{ }^{111} \mathrm{In}\right]-\mathrm{DTPA}$ EVs) per animal. After whole-body in vivo imaging analysis at $30 \mathrm{~min}-1 \mathrm{~h}, 4 \mathrm{~h}$, and $24 \mathrm{~h}$, mice were sacrificed, and perfused with saline, and organs were excised for quantitative analysis. Values were normalized to the organs and are expressed as mean $\pm \mathrm{SEM}$, where $\mathrm{n}=3$ for each group. Two-way ANOVA with Tukey's multiple comparison test. $* * * * p$-value $<0.0001,{ }^{*} p$-value $<0.05$. (B) Quantitative organ biodistribution of $\left[{ }^{111} \mathrm{In}\right]-\mathrm{DTPA}$ EVs in wild-type mice (without tumors). Mice were 
intravenously injected with $1 \times 10^{10}$ vesicles $\left[{ }^{111} \mathrm{In}\right]$-DTPA EVs and culled at $1 \mathrm{~h}, 4 \mathrm{~h}$ and $24 \mathrm{~h}$ post-injection. Animals were perfused with saline and their organs were excised for analysis by gamma counting. Values were either normalized to grams of tissue (left panel) or shown as per organ (right panel), and expressed as mean \pm SEM, where $\mathrm{n}=3$ for each group.

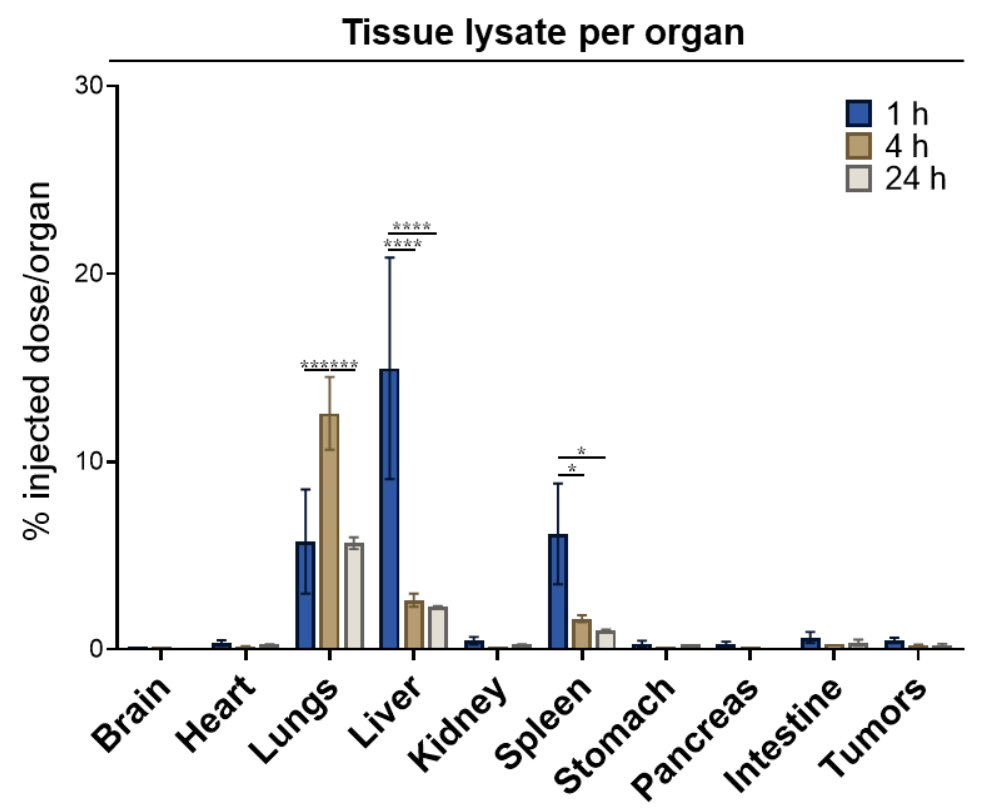

Figure S7. In vivo evaluation of NanoLuc Expi293F EV biodistribution by measuring bioluminescence in tissue lysates. CT26 tumor-bearing BALB/c mice were injected intravenously with $1 \times 10^{11}$ NanoLuc EVs via the tail vein. Organs were excised at $1 \mathrm{~h}, 4 \mathrm{~h}$ and $24 \mathrm{~h}$, homogenized using a lysis buffer and cleared of tissue debris before bioluminescence quantification on a FLUOstar ${ }^{\circledR}$ Omega plate reader. NanoLuc EV uptake in tissues was expressed as percentage injected dose per organ. Values are presented as mean \pm SEM, where $n=3$ for each group. Two-way ANOVA with Tukey's multiple comparisons test. ${ }^{* * * *} p$-value $<0.001,{ }^{* * *} p$-value $<0.005,{ }^{*} p$-value $<0.05$. 
A

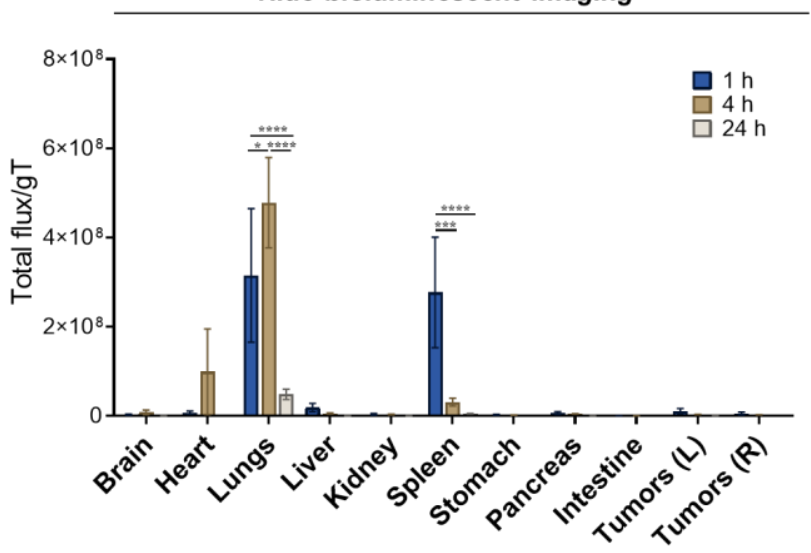

B

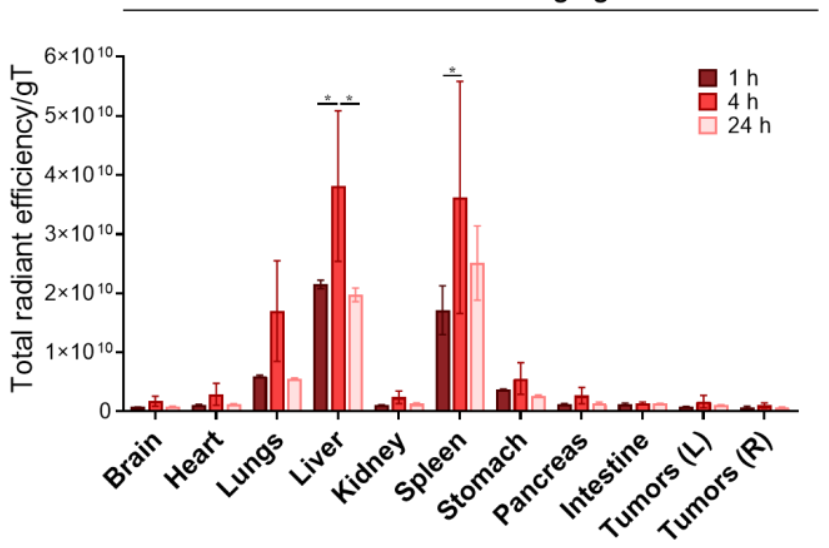

Figure S8. In vivo dual imaging biodistribution analysis of DiR-labeled NanoLuc EVs. Subcutaneous CT26 tumor-bearing BALB/c mice were injected intravenously with 1 x $10^{11}$ DiR-labeled NanoLuc (Nluc) EVs via the tail vein. Mice were culled at $1 \mathrm{~h}, 4 \mathrm{~h}$ and $24 \mathrm{~h}$ post-administration, perfused with saline, and the organs (brain, heart, lungs, liver, spleen, kidneys, pancreas, stomach, intestine, and tumors right (R) and left (L)) were excised. Organs were subjected to bioluminescence imaging for Nluc signals following immersion in the substrate furimazine for 30 sec, blotted on tissue paper, and imaged within $2 \mathrm{~min}$, and fluorescence imaging for near-infrared DiR signals. (A) Semi-quantitative analysis of Nluc signal from ex vivo images of whole-organs analyzed using the Living Image 4.7.2 software. Values are normalized to organ weight as total flux per grams of tissue (gT). (B) Semi-quantitative fluorescence analysis of the DiR signal from ex vivo imaging of DiR-labeled Nluc EV-treated mice. Individual ROIs were drawn for each organ to obtain their respective DiR fluorescence signals. Background signals from the PBStreated mice were subtracted from the data. Fluorescent signal is represented as total radiant efficiency $[\mathrm{p} / \mathrm{s}] /\left[\mu \mathrm{W} / \mathrm{cm}^{2}\right]$ per gT Data was analysed using the Living Image ${ }^{\circledR}$ 4.7.2 software. Values are expressed as mean \pm SEM. $N=3$ for all groups. Two-way ANOVA with Tukey’s multiple comparisons test. ${ }^{* * * *} p$ value $<0.001$, ${ }^{* * *} p$ value $<0.005$, ${ }^{*} p$ value $<0.05$. 